\title{
Ganadería familiar en el centro poblado de Santa Bárbara de Moro, distrito de Paucarcolla - Puno Perú
}

\author{
Family livestock in the populated center of Santa Bárbara de Moro, district of \\ Paucarcolla - Puno Peru
}

\author{
${ }^{1}$ Fermin Francisco Chaiña Chura \\ ${ }^{2}$ Juan Inquilla Mamani
}

ORCID: 0000-0003-0559-9748

ORCID: 0000-0003-2540-9091

\section{RESUMEN}

El objetivo de la investigación fue determinar la relación entre las variables socioeconómicas y la situación de la ganadería familiar de vacunos. En este tipo de ganadería, a pesar de que Puno sea considerado como el primer productor de ganado dentro de los departamentos de las zonas altoandinas del Perú, las unidades familiares se han visto afectadas por muchos factores que repercuten sobre los parámetros productivos y reproductivos y, por ende, en la economía del productor, manteniendo niveles de pobreza altos aún no evaluados en la zona. Bajo un diseño de investigación correlacional, explicativa y a nivel micro, se hizo el estudio exhaustivo aplicando la técnica de encuesta a 87 jefes de familia de una población de 109 familias. Los principales resultados evidenciaron que las variables socioeconómicas composición familiar, tenencia de tierra e ingreso tienen relación con la situación de la ganadería familiar de vacunos, que comprende a las variables tenencia de ganado vacuno, vacas en producción, producción de leche por vaca por día y destino de la producción de leche al mercado. Las variables sexo, edad y nivel educativo no tienen relación con la ganadería familiar de vacunos.

Palabras clave: Aspectos socioeconómicos, ganadería familiar, producción, vacunos.

\section{ABSTRACT}

The objective of the research was to determine the relationship between socioeconomic variables and the situation of family cattle raising. In this type of livestock, despite the fact that Puno is considered the first producer of cattle within the departments of the high Andean areas of Peru, family units have been affected by many factors that have an impact on the productive and reproductive parameters and, therefore, in the economy of the producer, maintaining high levels of poverty not yet evaluated in the area. Under a correlational, explanatory and micro-level research design, the exhaustive study was carried out applying the survey technique to 87 heads of families from a population of 109 families. The main results showed that the socioeconomic variables family composition, land tenure and income are related to the situation of the cattle raising, which includes the variables of cattle, cattle in production, milk production per cow per day and destination of milk production to the market. The variables sex, age and educational level are not related to the cattle raising.

Keywords: Socio-economic aspects, family stockbreeding, production, cattle.

Facultad de Ciencias Sociales. Universidad Nacional del Altiplano- Puno.E-mail: fchaina1@hotmail.com

Facultad de Ciencias Sociales. Universidad Nacional del Altiplano- Puno. 


\section{INTRODUCCIÓN}

La agricultura familiar a nivel mundial es pilar fundamental de los sistemas agroalimentarios, concordante con Requelme et al. (2016), en un contexto global de cambios científicos, tecnológicos y de comunicación en el sistema mundo, que ha provocado cambios en los sistemas de crianza de ganado vacuno en América Latina y en el Perú. Foronda (2004) considera que la crianza de vacunos no solo proporciona carne, sino también productos lácteos, pieles y energía para el arado. Por su parte, Cesín et al. (2007) precisa que la ganadería lechera es una actividad importante debido a que las condiciones agroecológicas permiten el cultivo de forrajes, además que los establos son pequeños y están adosados a la vivienda del productor. Se puede afirmar que la ganadería ha evolucionado y está en una etapa crucial de transición y cambio en estructura, manejo y gestión(García, 2014).

En el altiplano puneño esta actividad está enmarcada en la siguiente problemática:

a) Carencia de un diagnóstico de la ganadería familiar en el ámbito de estudio, en perspectiva, de realizar el análisis de la situación de esta actividad en este contexto.

b) Existencia de iniciativas individuales de innovación en el manejo, mejoramiento, sanidad y alimentación, que no permiten avanzar desde la visión de los jefes de familia y resulta necesaria la articulación productor-sector institucional.

c) No hay uniformidad en los sistemas de producción, las unidades familiares presentan particularidades de mejora en el funcionamiento de su sistema de producción familiar, principalmente en la crianza del ganado vacuno.

d) Los niveles de progreso no han sido reportados a nivel de investigación.

Es por estas razones que se planteó la presente investigación, para determinar la relación entre las variables socioeconómicas y la situación de la ganadería familiar de vacunos; cuya hipótesis fue: las variables socioeconómicas tienen relación con la situación de la ganadería familiar de vacunos en Moro.

En consecuencia, esta investigación analiza las características de la estructura y funcionamiento de estas unidades familiares en esta parte de los Andes, dedicadas a la crianza de ganado en el ámbito de estudio de la región de Puno; específicamente las variables sociales y económicas y la ganadería familiar, principalmente vacunos (Aubron y Cochet, 2008).

El conocimiento de la naturaleza de la economía familiar en la zona de estudio crea condiciones favorables para que el sector institucional pueda emprender acciones de intervención, a fin de impulsar el desarrollo local, por ende, el desarrollo regional y nacional. Los resultados de esta investigación contribuirán también a las actividades académicas en la universidad, por consiguiente, beneficiará a la sociedad en su conjunto.

\section{MATERIAL Y MÉTODOS}

Esta investigación se realizó en el centro poblado de Santa Bárbara de Moro, ubicado en la zona noreste del distrito de Paucarcolla, Región Puno, dedicada principalmente a la actividad pecuaria, en una altitud de $3812 \mathrm{msnm}$; costado este de la carretera panamericana Puno-Juliaca.

La investigación es de tipo correlacional y explicativa, debido a que se analizó la situación de la ganadería familiar de vacunos; desarrollada a nivel micro. Se realizó el estudio exhaustivo de un caso concreto en el ámbito de estudio. De una población de 109 familias (INEI, 2017), se determinó intencionalmente una muestra no probabilística, seleccionando arbitrariamente a 87 jefes de familia, en base a los criterios: ser criador de ganado vacuno, producir para el consumo de la familia y para el mercado, y que brinde información que se requiera.

La investigación se desarrolló utilizando métodos cuantitativos en la descripción y comprensión de la estructura y funcionamiento de las unidades familiares en el desarrollo de la crianza de ganado vacuno; la unidad de análisis fue la situación de la ganadería familiar de vacunos; la unidad de observación estuvo constituida por los jefes de familia.

Las variables de estudio fueron a) variables socioeconómicas, b) situación de la ganadería familiar, c) relación entre variables socioeconómicas y situación de la ganadería familiar de vacunos. Se empleó la metodología cuantitativa, utilizando la técnica de la encuesta y como instrumento el cuestionario, complementado con la observación, el análisis documental, entre otros, a fin de conocer la situación de la ganadería familiar; la verificación de las hipótesis se realizó con el apoyo de la 
prueba estadística de chi-cuadrada $\left(\mathrm{X}^{2}\right)$ (Amaya, 2017).

\section{RESULTADOS Y DISCUSIÓN}

La información obtenida se presenta en tres partes: la primera son las variables socioeconómicas, la segunda la ganadería familiar y finalmente la relación entre variables socioeconómicas y la situación de la ganadería familiar de vacunos.

\section{Variables socioeconómicas}

Están constituidas a su vez por variables sociales como edad, sexo, tamaño de la familia, nivel educativo; y variables económicas como tamaño del predio y el ingreso familiar por mes.

Tabla 1. Características de las variables socioeconómicas

\begin{tabular}{|c|c|c|c|c|c|c|c|c|c|}
\hline $\begin{array}{l}\text { Medidas } \\
\text { estadísticas }\end{array}$ & Edad & Sexo* & $\begin{array}{l}\text { Tamaño } \\
\text { de la } \\
\text { familia }\end{array}$ & $\begin{array}{c}\text { Nivel } \\
\text { educativo } \\
\text { (años) }\end{array}$ & $\begin{array}{l}\text { Tamaño } \\
\text { Predio } \\
\text { (há) }\end{array}$ & $\begin{array}{l}\text { Ingreso } \\
\text { mes } \\
(\mathrm{s} /)\end{array}$ & $\begin{array}{l}\text { Tenencia } \\
\text { ganado } \\
\text { vacuno }\end{array}$ & $\begin{array}{l}\text { Vacas } \\
\text { en } \\
\text { produc. }\end{array}$ & $\begin{array}{c}\text { Prod } \\
\text { leche/ } \\
\text { día/fam.(Lt.) }\end{array}$ \\
\hline Media & 48.03 & 00.00 & 4.08 & 8.85 & 3.02 & 585.86 & 11.83 & 5.70 & 38.54 \\
\hline Mediana & 47.00 & 1.00 & 4.00 & 11.00 & 1.00 & 400.00 & 10.00 & 5.00 & 20.00 \\
\hline Moda & 35 & 1 & 4 & 6 & 1 & 200 & 8 & 3 & 20 \\
\hline Des. estándar & 14.89 & 0.82 & 1.90 & 3.823 & 4.82 & 597.29 & 9.47 & 4.49 & 62.26 \\
\hline Mínimo & 18 & 1 & 1 & 1 & 0 & 0 & $3 * *$ & $0 * *$ & $0 * *$ \\
\hline Máximo & 85 & 2 & 12 & 16 & 30 & 3000 & 55 & 20 & 350 \\
\hline
\end{tabular}

*: La variable sexo está constituida por 56 hombres y 31 mujeres; **: existen pobladores que no crían ganado Fuente. Elaborado en base a la información del cuestionario.

En la Tabla 1 se observa que la edad promedio de los jefes de familia es de 48 años, con una variación de 18 a 85 años; la edad es una variable muy importante a la hora de entender la dinámica interna de la explotación, siendo las explotaciones de los ganaderos jóvenes los que han obtenido mejores resultados económicos, concordante con Bernúes (2007), Sin embargo, difieren de Paredes y Escobar- Mamani (2018), quienes encontraron que la edad es una variable que no resulta tener efecto significativo sobre la variable socioeconómicas como la pobreza.

La variable sexo es importante en el hacer y para conocer la situación de la ganadería familiar en Moro. En el estudio, participaron 56 varones y 31 mujeres, que representan el $64.4 \%$ y $35.6 \%$, respectivamente. Según Paredes y EscobarMamani (2018), la variable sexo tiene incidencia en la probabilidad de influir sobre la variable pobreza, al encontrar diferencias significativas $(p>0.05)$ a favor del sexo femenino como jefe de familia sobre la variable socioeconómica de pobreza, indicando que los hogares donde el jefe de hogar es hombre tienen mayor probabilidad de ser pobres, con respecto a los jefes de hogar liderado por mujeres.
La composición familiar constituye un componente muy importante en la organización social, base de la estructura familiar, y que presentan las siguientes características: El tamaño promedio de la familia es de 4 miembros, lo que indica que tienen entre 2 y 3 hijos, predominando familias con 3 y 4 integrantes. El número de integrantes de la familia es importante como perceptores de ingresos económicos con incidencia sobre variables socioeconómicas (pobreza), como lo refieren Paredes y EscobarMamani (2018).

En el trabajo ganadero, se observa que la especialización femenina, en la zona de estudio, vincula a las mujeres más a la producción de leche, concordante con Aubron y Cochet (2008) o Requelme et al. (2016). Este es el caso de las mujeres, quienes se han quedado a cargo de las unidades de producción y de los hijos que las apoyan (Jiménez et al., 2008).

La mano de obra familiar es importante para el desarrollo de la actividad ganadera (Vilaboa et al., 2012); debido a que estas unidades familiares de producción no contratan mano de obra, sino que movilizan el trabajo familiar en sus predios, concordante con De Torres et al. (2015). Todas las 
actividades se realizan principalmente con mano de obra familiar (Jiménez et al., 2008); la mano de obra para el ordeño es completamente familiar (Florio et al., 2012). En otros casos, esta forma de producción en la que la mayor parte del trabajo es realizado por los productores y sus familias, la categoría salario no desplaza al trabajo familiar (Albanesi, 2002).

El nivel educativo referido al número de años de escolaridad de educación formal logrados por el jefe de familia es un factor muy valioso en el desarrollo social y económico; los niveles educativos altos contribuyen al desarrollo sostenido en las capacidades humanas, concordante con Paredes y Escobar-Mamani (2018). Se presenta predominante los niveles educativos de primaria y secundaria en el $43.7 \%$ y $40.2 \%$ respectivamente; seguido de educación superior con el $10.3 \%$ y el $4.6 \%$ de la población que deja de estudiar o no concurre a un centro de educación formal; el promedio del nivel de escolaridad de los jefes de familia es de 8.85 años de educación formal.

En promedio las unidades familiares poseen 12 vacunos entre adultos y crías, entre vacas en producción y vacas que están en seca (vacas preñadas en los dos últimos meses de gestación). De la información obtenida, mediante la encuesta a nivel familiar, el promedio de producción de leche es de 39 litros por día, en base a un promedio de 6 vacas en producción que equivale a una producción vaca/día de $6.5 \mathrm{~L}$, que se considera dentro del nivel de producción de la zona donde se encuentran factores limitantes de pastos en cantidad y calidad, así como las condiciones climáticas como lo refieren Gil y Santos (2006). En la zona de estudio existe vocación de crianza de ganado vacuno como sucede en el resto del país, experiencia lograda desde el reparto de tierras por la reforma agraria y que posteriormente se integra constituyendo la Sociedad Agrícola de Interés Social - SAIS Buena Vista. Y esta, posteriormente, al desintegrarse por la reestructuración agraria, en parte, se adjudica a la Comunidad Campesina de Santa Bárbara de Moro y a su vez se parcela entre sus socios por acuerdo de Asamblea General. Respecto al número de cabezas de ganado este no influye sobre el aspecto socioeconómico como lo reportan Paredes y Escobar-Mamani (2018).
Referente al tamaño del predio, la población del ámbito de estudio tiene tamaños de predios familiares diferentes; la tenencia del recurso tierra es un medio de producción básica y fundamental para la actividad de crianza de vacunos y agricultura de cultivos andinos. Se presenta predominante los predios de 1 y 2 hectáreas en una proporción de $55.2 \%$ y $18.4 \%$ respectivamente; seguido de predios de 3,4 y 5 hectáreas en una proporción de $8.0 \%, 3.4 \%$ y $3.4 \%$, y es notorio la tenencia de propiedades de 12 y 15 hectáreas que representa al $3.4 \%$ y $2.3 \%$ respectivamente; siendo el predio de mayor dimensión uno de 30 hectáreas. Los productores de esta zona cuentan con varias parcelas, cuya suma conforma el tamaño promedio de 3.02 hectáreas, entre pastos naturales, cultivados y áreas para cultivos andinos.

Al respecto, Cervantes y Cesin (2007) considera que las unidades de producción utilizan recursos de origen familiar, en especial: tierra, agua y capital, semejante a lo que sucede en la zona de estudio. Para Gazzano y Achkar (2016), el territorio emerge de la interacción de componentes de la naturaleza en sus dimensiones física, biológica, productiva y tecnológica, producto de una determinada organización social, política, económica y cultural.

El ingreso económico mensual varía entre los que no tienen ingresos por mes. El mínimo es de $\mathrm{S} / 20$ en el nivel inferior y el máximo es de $\mathrm{S} / 3000$ al mes. Asimismo, se observa una relevancia en el grupo que fluctúa de $\mathrm{S} / 21$ y S/ 520; seguido por el grupo comprendido entre $\mathrm{S} / 521$ y S/ 1020 que representan el $52.8 \%$ y $27 \%$ respectivamente, que en conjunto constituyen el $89.8 \%$; y otros grupos con mayores ingresos representan el 11.2 $\%$, cuyos ingresos varían entre S/ 1021 y S/ 3000 ; resultando el promedio de ingreso familiar por mes de $\mathrm{S} / 585.86$.

En las unidades familiares se observa que, a pesar de contar con una parte de ganado especializado en la producción de leche, esta es baja, la cual se ve limitada por la alimentación. Por lo tanto, solo se realiza un ordeño diario, dejando un cuarto de la ubre para la alimentación del ternero; sin embargo, esto permite mejorar el crecimiento del ternero, lo cual es concordante con Martinez et al. (2012). 
Tabla 2. Ingreso familiar por mes en soles

$\begin{array}{ccccc}\text { Niveles de ingreso (S/) } & \text { Frecuencia } & \text { Porcentaje } & \begin{array}{c}\text { Porcentaje } \\ \text { válido }\end{array} & \begin{array}{c}\text { Porcentaje } \\ \text { acumulado }\end{array} \\ <20 & 6 & 6.7 & 6.9 & 6.9 \\ 21-520 & 47 & 52.8 & 54.0 & 60.9 \\ 521-1020 & 24 & 27.0 & 27.6 & 88.5 \\ 1021-1520 & 5 & 5.6 & 5.7 & 94.3 \\ 1521-2020 & 3 & 3.4 & 3.4 & 97.7 \\ 2521> & 2 & 2.2 & 2.3 & 100.0 \\ \text { Total } & 87 & 97.8 & 100.0 & \end{array}$

Se construye una lógica económica alrededor de la producción de leche en los sistemas de producción, en la cual la venta regular de queso permite comprar los alimentos de la familia para la semana, como lo sostienen Aubron y Cochet (2008). La lechería familiar se transforma en una estrategia muy importante para buscar la reducción de la pobreza y aumentar la seguridad alimentaria de las familias; se debe a que es una actividad económica muy estable con ingresos periódicos, a veces cada semana o todos los días (Cervantes y Cesin, 2007). Todo esto dependiendo del tipo de comercialización y producto, que permite el acceso a niveles de confort básicos en vivienda, agua y energía, lo que constituye un rasgo importante para el estímulo a la permanencia en el medio rural (García et al., 2011).

Al respecto, Albanesi (2007) considera que la producción familiar se vincula con la economía global en cada tiempo y espacio; sin embargo, es necesario reforzar las capacidades de gestión de los recursos disponibles para alcanzar el desarrollo sustentable como lo indica Álvarez et al. (2018), y aprovechar los fuertes vínculos que tienen sus productos con la cultura gastronómica local, a fin de obtener ingresos familiares (Cesín et al., 2007; Jiménez et al., 2014)

\section{Situación de la ganadería familiar}

En la Tabla 3 y figuras 1 y 2 se observa la tenencia de ganado vacuno con un mínimo de 3 y un máximo de 55 cabezas de ganado. Resultan relevantes dos grupos, entre el $51.7 \%$ y el $20.2 \%$ de productores cuentan con un hato que varía de 6 a 15 y 16 a 25 unidades respectivamente; es importante también el grupo que cuenta con 3 a 5 vacunos que representa el $21.3 \%$ de criadores.

Los productores del ámbito tienden a mejorar la ganadería familiar de vacunos con la adquisición de ganado Brown Swiss, que suele desplazar al ganado nativo, concordante con lo que señalan Álvarez et al. (2004), García (2014) y Sabourin et al. (2015). La alimentación se da en base a pastos naturales y cultivados como la alfalfa verde en pastoreo extensivo predominantemente; lascondiciones agroecológicas necesarias para la producción de forrajes demanda humedad (Cesín et al., 2007); que se presenta en la época de temporal, situación que aprovechan los habitantes para instalar pastos cultivados para desarrollar una ganadería lechera familiar más sustentable.

Tabla 3. Tenencia de ganado vacuno

\begin{tabular}{|ccccc|}
$\begin{array}{c}\text { Niveles de } \\
\text { tenencia ganado }\end{array}$ & Frecuencia & Porcentaje & $\begin{array}{c}\text { Porcentaje } \\
\text { válido }\end{array}$ & $\begin{array}{c}\text { Porcentaje } \\
\text { acumulado }\end{array}$ \\
$<5$ & 19 & 21.3 & 21.8 & 21.8 \\
$6-15$ & 46 & 51.7 & 52.9 & 74.7 \\
$16-25$ & 18 & 20.2 & 20.7 & 95.4 \\
$26-35$ & 1 & 1.1 & 1.1 & 96.6 \\
$36-45$ & 1 & 1.1 & 1.1 & 97.7 \\
$46+$ & 2 & 2.2 & 2.3 & 100.0 \\
Total & 87 & 97.8 & 100.0 & \\
\hline
\end{tabular}




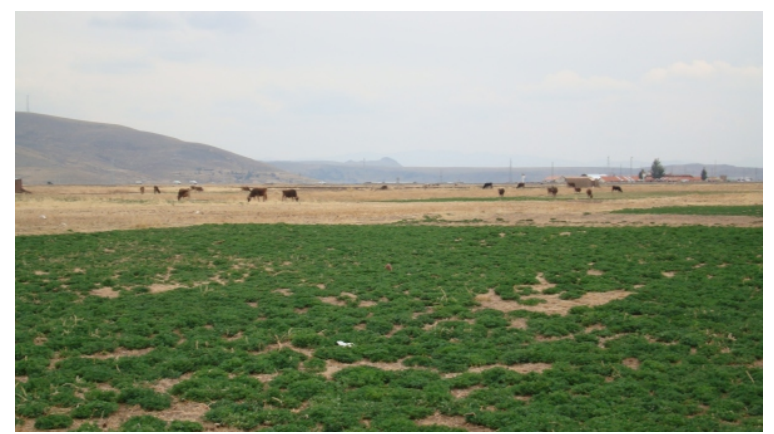

Figura 1. Ganadería familiar C.P. Accaso

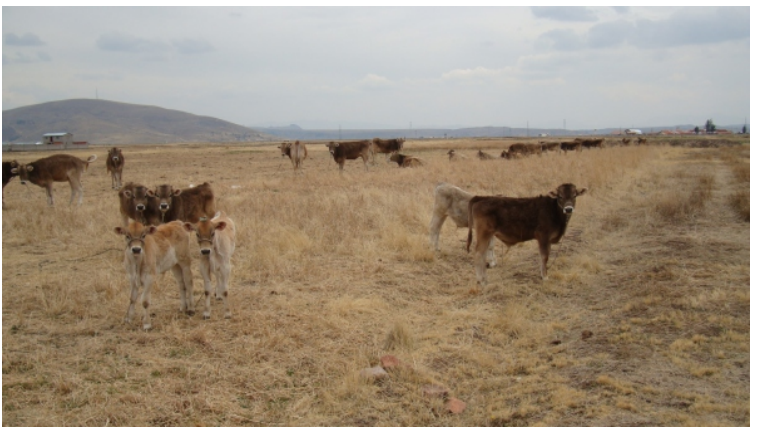

Figura 2. Ganadería familiar C.P. Moro

Para Moreno et al. (2012), los sistemas familiares de bovinos productores de leche son considerados como sistemas en paralelo; es decir, en simbiosis entre hombre y animal, relación que solo es afectada por factores ambientales, semejante a lo que se observa en la presente investigación.

Las principales características de la lechería familiar se centran en:

a) Su conformación socioeconómica, determinada por la estructura de las zonas rurales del país.

b) Uso de recursos tales como mano de obra familiar, cultivos forrajeros y residuos de cosecha producidos en sus parcelas, con una reducida inversión en insumos.

c) Poca inversión en infraestructura.

d) El manejo del hato se da en condiciones de estabulación o semiestabulación, en instalaciones cercanas a la vivienda de la familia (Sánchez y Sánchez 2005 citado por Moreno et al., 2012); las instalaciones se localizan en el predio donde se encuentra la vivienda (Jiménez et al., 2008); en otros casos los pequeños productores tienen limitada vinculación al mercado (Cavallotti, 2014).

En las figuras 1 y 2 se observa la existencia de ganado vacuno raza Brown Swiss mejorado y criollo. La zona de Moro ofrece condiciones favorables para producir leche orgánica, principalmente por los conocimientos ancestrales y tecnologías de producción en la agricultura y ganadería, que aún se mantiene y se orienta al cuidado de los recursos naturales, lo que garantiza una certificación orgánica en el menor tiempo posible, debido a la ventaja tradicional de producir casi sin químicos, situación que es factible de aprovechar en beneficio de la ganadería familiar, como lo refieren Olarte y Olarte (2013).

Las cadenas lecheras de transformación y de comercialización se dan de la siguiente manera:

El primer circuito artesanal es organizado alrededor de la venta de leche cruda por parte de los productores establecidos en la cercanía de las ciudades; en el segundo, los ganaderos transforman la leche en productos procesados como el queso que venden regularmente a negociantes intermediarios, quienes lo transportan hasta los mercados urbanos; la tercera cadena artesanal corresponde a las pequeñas queserías que acopian leche a nivel local y se encargan de la transformación y de la comercialización de sus productos como lo refieren Aubron y Cochet (2008).

\section{Relación entre variables socioeconómicas y situación de la ganadería familiar}

La verificación de la hipótesis se realiza en base a la información obtenida en el ámbito de investigación, vía cuestionario; se postula que: Las variables sociales y económicas tienen relación con la situación de la ganadería familiar: vacunos en el centro poblado de Santa Bárbara de Moro.

La hipótesis estadística:

Ho: Las variables sociales son independientes de la situación de la ganadería de vacunos a nivel familiar.

Ha: Las variables sociales no son independientes de la situación de la ganadería de vacunos a nivel familiar.

El análisis se realizó con la verificación de las hipótesis estadísticas, a través de la prueba de chicuadrada de Pearson, lo que permite determinar si existe relación entre las variables. Los resultados fueron: 
Tabla 4. Variable composición familiar y tenencia de ganado vacuno a nivel familiar

\begin{tabular}{lccc|} 
& Valor & gl & $\begin{array}{c}\text { Significación } \\
\text { asintótica (bilateral) }\end{array}$ \\
Chi -cuadrado de Pearson & $334.125^{\text {a }}$ & 270 & .005 \\
Razón de verosimilitud & 170.694 & 270 & 1.000 \\
Asociación lineal por lineal & 1.715 & 1 & .190 \\
N de casos válidos & $8 \mathrm{~m} 7$ & &
\end{tabular}

Fuente. Elaborado en base a la información del cuestionario y prueba chi-cuadrado.

En la tabla 4 se observa que el valor de la significación asintótica es menor a 0.05 , que indica que existe asociación entre la variable composición familiar y tenencia de ganado vacuno a nivel familiar; por lo que se acepta la hipótesis alterna. El rol del jefe de familia es importante en la crianza de vacunos, sea el padre o la madre, ya que es responsable del manejo, alimentación, sanidad y reproducción del ganado de la unidad de producción familiar, pero obtiene resultados productivos bajos. Sin embargo, PicaCiamarra (2005) considera que, a pesar de las políticas de aumento de la producción, estas no benefician necesariamente a los ganaderos pobres de las unidades familiares, que son un grupo heterogéneo y en muchos casos extremadamente pobres, ya que muchos de ellos maximizarían una supervivencia más que una función de producción.

En la Tabla 5 se presenta los resultados de la prueba chi-cuadrada para verificar el nivel de relación entre las variables sociales y la situación de la ganadería familiar.

Tabla 5. Variables sociales y tenencia de ganado vacuno a nivel familiar

\begin{tabular}{|c|c|c|c|}
\hline Chi- cuadrado de Pearson y variables & Valor & gl & $\begin{array}{l}\text { Significación } \\
\text { asintótica } \\
\text { (bilateral) }\end{array}$ \\
\hline \multicolumn{4}{|l|}{ Chi-cuadrado de Pearson: } \\
\hline 1. Sexo y tenencia ganado vacuno & $23.245^{\mathrm{a}}$ & 27 & .672 \\
\hline 2. Sexo y vacas en producción de leche & $6.834^{\mathrm{a}}$ & 13 & .911 \\
\hline 3. Sexo y producción de leche/vaca/día & $19.314^{\mathrm{a}}$ & 16 & .253 \\
\hline 4. Sexo y total de producción de leche/día/fam. & $38.478^{\mathrm{a}}$ & 33 & .235 \\
\hline 5. Sexo y destino producción leche/mercado & $50.096^{\mathrm{a}}$ & 43 & .213 \\
\hline 6. Edad y tenencia ganado vacuno & $1148.523^{\mathrm{a}}$ & 1080 & .072 \\
\hline 7. Edad y vacas en producción de leche & $542.357^{\mathrm{a}}$ & 520 & .241 \\
\hline 8. Edad y producción de leche/vaca/día & $577.790^{\mathrm{a}}$ & 640 & .962 \\
\hline 9. Edad y total de producción de leche/día/fam. & $1356.651^{\mathrm{a}}$ & 1320 & .236 \\
\hline 10 Edad y destino producción leche/mercado & $1802.937^{\mathrm{a}}$ & 1720 & .080 \\
\hline 11. C.f.* y tenencia ganado vacuno & $334.125^{\mathrm{a}}$ & 270 & .005 \\
\hline 12. C.f. y vacas en producción de leche & $159.544^{\mathrm{a}}$ & 130 & .040 \\
\hline 13. C.f. y producción de leche/vaca/día & $208.173^{\mathrm{a}}$ & 160 & .006 \\
\hline 14. C.f. y total de producción de leche/día/fam. & $303.896^{\mathrm{a}}$ & 330 & .846 \\
\hline 15. C.f. y destino producción leche/mercado & $500.711^{\mathrm{a}}$ & 430 & .010 \\
\hline 16. $\mathrm{NE}^{* *}$. y tenencia ganado vacuno & $124.955^{\mathrm{a}}$ & 108 & .126 \\
\hline 17. NE. y vacas en producción de leche & $69.502^{\mathrm{a}}$ & 52 & .053 \\
\hline 18. NE. y producción de leche/vaca/día & $74.527^{\mathrm{a}}$ & 64 & .173 \\
\hline 19. NE. y total de producción de leche/día/fam. & $155.061^{\mathrm{a}}$ & 132 & .083 \\
\hline 20. NE. y destino producción leche/mercado & $176.274^{\mathrm{a}}$ & 172 & .396 \\
\hline $\mathrm{N}^{0}$ de casos válidos & 87 & & \\
\hline
\end{tabular}

C.f*. $=$ Composición familiar; NE $* *=$ Nivel educativo

Fuente. Elaborado en base a la información del cuestionario y prueba chi-cuadrado de Pearson. 
En la Tabla 5 se observa que, de las 20 variables solo 4 son representativas con valor de significancia menor a 0.05 . Al cumplir este parámetro, la variable composición familiar tiene relación con tenencia de ganado vacuno, vacas en producción de leche, producción de leche por vaca/día y destino de la producción de leche/mercado; en los otros casos no hay relación significativa entre variables.

La hipótesis estadística:

Ho: Las variables económicas son independientes de la situación de la ganadería de vacunos a nivel familiar.
Ha: Las variables económicas no son independientes de la situación de la ganadería de vacunos a nivel familiar.

En la Tabla 6, al aplicar la prueba chi-cuadrada para verificar el nivel de asociación existente entre las variables económicas y la situación de la ganadería familiar, se observa que de las diez variables solo cuatro son representativas con valor de asociación significativa, dado que el valor es menor a $0.05, \mathrm{Al}$ cumplir este parámetro, las variables tenencia de tierra e ingreso tiene relación con tenencia de ganado vacuno y producción de leche por vaca por día; en los otros casos no hay relación entre variables.

Tabla 6. Variables económicas y tenencia de ganado vacuno a nivel familiar

\begin{tabular}{|c|c|c|c|}
\hline Chi - cuadrado de Pearson y variables & Valor & gl & $\begin{array}{c}\text { Significación } \\
\text { asintótica } \\
\text { (bilateral) }\end{array}$ \\
\hline 1. Tt.* y tenencia ganado vacuno & $492.024^{\mathrm{a}}$ & 297 & .000 \\
\hline 2. Tt. y vacas en producción de leche & $181.408^{\mathrm{a}}$ & 143 & .016 \\
\hline 3. Tt. y producción de leche/vaca/día & $227.957^{\mathrm{a}}$ & 176 & .005 \\
\hline 4. Tt. y total de producción de leche/día/fam. & $336.633^{\mathrm{a}}$ & 363 & .836 \\
\hline 5 Tt. y destino producción leche/mercado & $544.488^{\mathrm{a}}$ & 473 & .013 \\
\hline 6. Ingreso y tenencia ganado vacuno & $785.350^{\mathrm{a}}$ & 648 & .000 \\
\hline 7. Ingreso y vacas en producción de leche & $318.710^{\mathrm{a}}$ & 312 & .385 \\
\hline 8. Ingreso y producción de leche/vaca/día & $434.009^{\mathrm{a}}$ & 384 & .040 \\
\hline 9. Ingreso y total de producción leche/día/fam. & $769.296^{\mathrm{a}}$ & 792 & .712 \\
\hline 10 Ingreso y destino producción/mercado & $1090.615^{\mathrm{a}}$ & 1032 & .100 \\
\hline $\mathrm{N}^{0}$ de casos válidos & 87 & & \\
\hline
\end{tabular}

Tt. $*=$ Tenencia de tierra.

Fuente: Elaborado en base a la información del cuestionario y prueba chi-cuadrado de Pearson.

En consecuencia, se evidencia que la variable composición familiar es asociada con tenencia de ganado vacuno, vacas en producción de leche, producción de leche por vaca por día y destino de la producción de leche/mercado; al igual que las variables tenencia de tierra, ingreso con tenencia de ganado vacuno y producción de leche vaca por día.

Evidenciándose que las variables socioeconómicas tienen relación diferenciada con la situación de la ganadería familiar de vacunos en elámbito de estudio.

Los resultados obtenidos difieren de lo reportado por Paredes y Escobar-Mamani (2018), quienes afirman que no existe dependencia del activo ganadero con variables sociales como la pobreza, en un estudio realizado en la zona rural de Puno; cuyos resultados sugieren que la pobreza monetaria, pobreza crónica y la pobreza reciente depende significativamente del número de miembros del hogar, el número de perceptores de ingreso en el hogar, el nivel de educación de los jefes de hogar, la carencia de servicios higiénicos en la vivienda, el número de hectáreas de tierra y el número de parcelas, entre otros.

Los resultados refieren la importancia de las unidades ganaderas familiares en la zona de estudio y hoy más que nunca merecen la debida atención para enmarcarlas dentro de la visión de desarrollo sustentable, como lo sugiere De Torres et al. (2015), al considerar que es posible la transformación del minifundio hacia paisajes agrarios más intensivos y productivos. Esto 
amerita un diseño de las políticas públicas no solo en materia de desarrollo rural, sino también sobre las políticas que suponen reconocimiento y mejora de las condiciones de vida (Rossi, 2010; De Torres et al.,2015).

Requelme et al. (2016) refiriere también que las explotaciones familiares son importantes sectores productivos que aportan a la vocación campesina en muchos países, por lo que es imprescindible involucrarlos en lo posible en la adopción de tecnologías e innovación, como mecanismos que mejoren sus niveles productivos y económicos, así como sus implicancias ambientales y sociales. Complementadas con la propuesta de Freire (1984), es decir, la vía de la comunicación: la dialógica; pues a través de comunicación de ida y vuelta (vía problematización entre los interesados), los productores y los técnicos del sector institucional es posible emprender acciones de desarrollo del medio rural, buscando la sustentabilidad.

\section{CONCLUSIONES}

Las variables socioeconómicas tienen relación o asociación significativa con la situación de la ganadería familiar de vacunos en la variable composición familiar asociada con tenencia de ganado vacuno, vacas en producción de leche, producción de leche por vaca por día y destino de la producción de leche/mercado; al igual que las variables tenencia de tierra e ingreso con tenencia de ganado vacuno, producción de leche por vaca por día.

En las variables sexo, edad y nivel educativo no se observó asociación con las 5 variables de la ganadería familiar.

La identificación de la situación de la ganadería familiar y de la asociación con las variables socioeconómicas permitirá el mejor uso de los recursos y plantear alternativas de mejora en beneficio de las condiciones de vida de las unidades familiares.

\section{REFERENCIAS}

AMAYA, L. Prueba Chi-cuadrado en la estadística no paramétrica. Revista de investigación de la Facultad de Ciencias, 1(1): 13-17, 2017.

ALBANESI, R. La modernización en el devenir de la producción familiar capitalizada. Mundo Agrario. Revista de estudios rurales, 7(14): 115, 2007.
ÁLVAREZ, G., HERRERA, J., BARCENA, R., MARTÍNEZ, F., HERNÁNDEZ, A., y PÉREZ, J. Calidad de la alimentación y rentabilidad de granjas lecheras familiares del sur del valle de México. Archivos de zootecnia, 53(201): 103-106, 2004.

Álvarez, J., PABlO, A., MOLINA, C., NORBERTO, R., y HEGEDUS, P. Caracterización del sistema de innovación para la ganadería familiar en la región de la sierra del Este de Uruguay. Rev. de investig. agroproducción sustentable, 2(3), $80-90$ $\begin{array}{llll}2 & 0 & 1 & 8\end{array}$. DOI: $10.25127 /$ aps.20182.396

AUBRON, C., \& COCHET, H. Producción lechera en los Andes peruanos: ¿Integración al mercado interno o marginación económica? Anuario americanista europeo, (6-7), 217 238, 2008.

BERNÚES, J. Ganadería de montaña en un contexto global: evolución, condicionantes y oportunidades. Centro de investigación y tecnología agroalimentaria de Aragón. Zaragoza España. Pastos. 37 (2): 133-175, 2007.

CAVALLOTTI, B. Ganadería bovina de carne y leche. Problemática y alternativas. El Cotidiano, (188): 95-101, 2014.

CERVANTES, F., y CESIN, A. La pequeña lechería rural o urbana en México y su papel en el amortiguamiento de la pobreza. Rev. Unell. Cienc. Tec. (25): 72-85, 2007.

CESÍN, Alfredo; ALIPHAT, Mario; RAMIREZ, Benito; HERRERA, José y MARTÍNEZ, Daniel. Ganadería lechera familiar y producción de queso. Estudio en tres comunidades del municipio de Tetlatlahuaca en el estado de Tlaxcala, México. Técnica pecuaria en México, 45(10): 61-76, 2007.

DE TORRES, M., PEDRO, A., ERIC, S., JOAQUIN, C., y GILLES, M. Uruguay: La agricultura familiar entre dos proyectos contrapuestos. En Eric, S., Samper, M., y Sotomayor, O. (Ed.). Políticas públicas y agricultura familiar en América Latina y el Caribe: nuevas perspectivas (319-339). San José, Costa Rica: Red políticas públicas y desarrollo rural en América Latina, Cirad, Cooperación regional 
francesa para América del Sur, CEPAL, IICA. 2015.

FREIRE, P. ¿Extensión o comunicación? la concientización en el medio rural. México: siglo XXI. 1984.

FLORIO, J., TAMASAUKAS, R., y RIVERA, S. Diagnóstico participativo de hemotrópicos en bovinos a nivel de peque ños productores y productoras de ganadería doble propósito en el Sur del Estado Aragua en la república bolivariana $\mathrm{d}$ e $\mathrm{Ven}$ e z u e $1 \mathrm{a}$. A c t as iberoamericanas de conservación animal, 2, 163-170.2012.

FORONDA, R. Promoción de inversiones en la ganadería familiar de las tierras bajas de Bolivia. Proyecto de cooperación técnica $\mathrm{FAO} / \mathrm{TCP} /$ 2910 apseyo a la integración agropecuaria en el MERCOSUR ampliado. FAO. Recuperado de promobol.pdf(página 1a 55). 2004.

GARCÍA, Anastasio; ALBARRÁN, Benito y AVILÉS, Francisca, Dinámicas y tendencias de la ganadería doble propósito en el sur del estado de México. Agrociencia, 49(2): 125$139,2014$.

GARCÍA, Rodrigo, DIEGUEZ, Francisco, MOLINA, Carlos, GUTIERREZ, Ramón y TOMMASINO, Humberto. Sustentabilidad de los criadores familiares. Rev. Ganaderos, decisiones, sustentabilidad y familia. 64-68, 2011.

GAZZANO, I., y ACHKAR, M. (2016). Conflictos de las transformaciones territoriales: Ganaderos frente a la intensificación agraria en Esteros de Farrapos Uruguay. Revista Iberoamericana de Economía Ecológica, 26, 109-121, 2016.

GIL, J y SANTOS, R. Plan orientador para la ganadería tropical en Pozuzo y Palcazú. Desarrollado en cumplimiento al convenio suscrito entre la Dirección General de Promoción Agraria del MINAG (DGPA), Programa de Desarrollo. Alternativo en las Áreas de Pozuzo y Palcazu (PRODAPP) y el Instituto Interamericano de Cooperación para la Agricultura (IICA). Lima-Perú. 2006.

Instituto Nacional de Estadística e Informática. Directorio Nacional de centro poblados. Censos Nacionales 2017. XII de población, VII de vivienda y II de comunidades indígenas. 2017.

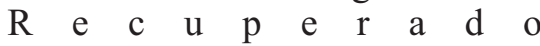
https://www.inei.gob.pe/media/Me nuRecursivo/publicaciones_digital es/Est/Lib1541/index.htm

JIMÉNEZ, F., PESADO, A., GARCÍA, A., DÁVALOS, J., ESPINOSA, V., y DUCOING, D. (2008). Persistencia de la lechería familiar en el municipio de Maravatío, Michoacán. Livestock Research for Rural Development, 20(10): 1-12, 2008

JIMÉNEZ, R., ESPINOZA, V., Y SOLER, D. El costo de oportunidad de la mano de obra familiar en la economía de la producción lechera de Michoacán, México. Revista de Investigación Agraria y Ambiental, 5(1), 47-56, 2014.

MORENO, A., HERRERA, G., CARRIÓN, M., ÁlVAREZ, D., PÉREZ, R., y ORTIZ, R. Caracterización y modelación esquemática de un sistema familiar de bovinos productores de leche en la Ciénega de Chapala, México. Archivos Latinoamericanos de Producción Animal, 20(3-4): 85-94, 2012.

MARTÍNEZ, C., y ABAD, J. Características de la producción y comercialización de leche bovina en sistemas de doble propósito en dobladero, Veracruz. Revista mexicana de agronegocios, 30(16): 816-824, 2012.

OLARTE, S., y OLARTE, U. La producción de leche orgánica en la región de Puno: una alternativa de desarrollo sostenible. Mundo Agrario, 13(26), 2013. ISSN 1515-5994.

PAREDES R, \& ESCOBAR-MAMANI, F. El rol de la ganadería y la pobreza en el área rural de Puno. Revista de Investigaciones Altoandinas, 20(1), $39-60, \quad 2018$. https://dx.doi.org/10.18271/ria.201 8.32 .

PICA-CIAMARRA, U. Livestock Policies for Poverty Alleviation: Theory and Practical Evidence from Africa, Asia and Latin America. Prop-poor Livestock Policy Iniciative. 2005.

REQUELME N.,AFONSO, A., DERBE, R., MENP MONCAYO, M., y ESCOBAR, J. Innovación en 
agricultura familiar: aplicación a sistemas de producción ganadero lechero en la sierra norte del Ecuador. 20th International Congress on Project Management and Engineering Cartagena, 20542066, 2016.

ROSSI, V. La producción familiar en la cuestión agraria uruguaya. Revista nera, 13(16), 63-80, 2010. ISSN: 18066755.

SABOURIN, E., DE TORRES, M., ARBELETCHE, P., COURDIN, V., FRANCOIS, J., y MORALES, H. Políticas públicas y ganadería familiar en Uruguay: los desafíos ambientales y de ordenamiento territorial. Pampa. Revista Interuniversitaria de Estudios Territoriales, año 11 (12), 241-264, 2015.

VILABOA, J., QUIRÓS, O., PABLO, D., CHING, R., BROWER, N., y ZETINA, P. Los sistemas ganaderos con criollo lechero tropical (reyna) en Costa Rica. Agronomía Mesoamericana, 23(1): 167-178, 2012. 